\title{
Resistencia a la presión de pares y pareja y consumo de tabaco y alcohol en adolescentes
}

\section{Resistance to peer and partner pressure and tobacco and alcohol use among adolescents}

Patricia Andrade Palos; Citlall Pérez de la Barrera; Lilia Bertha Alfaro Martínez; Martha Elba SáncheZ Oviedo; Alicia López Montes de Oca
| Universidad Nacional Autónoma de México

Enviar correspondencia a:

Patricia Andrade Palos. Facultad de Psicología, Avenida Universidad 3004 Col. Copilco-Universidad. CP 04510 México DF.

Correo electrónico: palos@servidor.unam.mx.

\section{RESUMEN}

Introducción: El consumo de drogas representa un problema de salud pública en México. En el ámbito internacional y desde la perspectiva de la promoción de la salud, destaca el enfoque de Habilidades para la vida propuesto por la Organización Mundial de la Salud, que identifica a la habilidad de resistencia a la presión como un componente clave para la prevención de consumo de drogas legales e ilegales en adolescentes.

Objetivo: se desarrolló y validó un instrumento para medir la habilidad de resistencia a la presión que permita confirmar si existen diferencias en la habilidad de resistencia a la presión en adolescentes no consumidores y consumidores de alcohol y tabaco como la evidencia empirica reporta.

Material y métodos: la muestra estuvo constituida por 5651 adolescentes, 2637 (47.9\%) hombres y 2864 (52.1\%) mujeres estudiantes de bachilleratos públicos de la Ciudad de México, seleccionados de manera aleatoria con una media de edad de 16.7 años. Se utilizó el instrumento validado en la primera fase del estudio. Para medir el patrón de consumo se utilizó una escala elaborada con base en los indicadores de la Encuesta Nacional de Adicciones (2002).

Resultados: El análisis factorial del instrumento arrojó tres factores: aceptación de la presión de amigos, aceptación de la presión de la pareja y resistencia a la presión de amigos y/o pareja. Los estudiantes no consumidores de alcohol y tabaco tienen mayor puntaje en la habilidad de resistencia a la presión a diferencia de los consumidores en el último mes.

Conclusiones: con base en estos resultados se pretende desarrollar un programa de prevención de adicciones para estudiantes de un bachillerato público de la Ciudad de México.

Palabras clave: resistencia a la presión, pares, adolescentes, alcohol, tabaco.

\section{ABSTRACT}

Introduction: Drug consumption constitutes a public health problem in Mexico. In the international literature and from the health promotion perspective, the Life Skills Approach proposed by the World Health Organization identifies the ability to resist pressure as a key component in the prevention of legal and illegal drug use among adolescents.

Objective: An instrument for measuring this ability was developed and validated in order to confirm whether, as the empirical evidence suggests, it differs between users and non-users of alcohol and tobacco.

Material and methods: The sample was made up of 5651 adolescents, 2637 $(47.9 \%)$ of whom were male and 2864 (52.1\%) female. These participants were selected at random from among public high school pupils in Mexico City, and average age was 16.7 years. The instrument used was that validated in the first phase of the study. To measure patterns of use, we used a scale based on indicators derived from the National Addiction Survey (2002).

Results: Factor analysis yielded three factors: peer pressure acceptance, partner pressure acceptance and peer/partner pressure resistance. Non-users of alcohol and tobacco scored higher in the ability to resist pressure than those who had used alcohol and tobacco in the last month.

Conclusions: Based on these results, the aim is to develop an addiction prevention program for public high school pupils in Mexico City.

Key words: resistance to pressure, peers, adolescents, alcohol, tobacco. 


\section{INTRODUCCIÓN}

En México, el consumo de drogas en adolescentes crece día a día: más de 200 mil adolescentes entre 12 y 17 años han usado drogas, especificamente se ha identificado un incremento en el consumo de alcohol, marihuana y metanfetaminas. No obstante que el índice general de consumo se mantiene estable, se observan cambios en la distribución de los índices de consumo de hombres y mujeres de manera que actualmente la prevalencia de consumo de alcohol y tabaco de las mujeres, se presenta con valores similares a la de los hombres (Encuesta Nacional de Adicciones, ENA, 2002).

Más recientemente, la encuesta sobre consumo de drogas en estudiantes del Distrito Federal, que se levantó en noviembre del 2006 (Villatoro, Gutiérrez, Quiroz, Moreno, Gaytán, Gaytán, et al., 2007) y donde participaron 10,523 adolescentes; $50.5 \%$ hombres y $49.5 \%$ mujeres, señala un incremento importante en el consumo de drogas en los últimos 3 años, que ha pasado del $15.2 \%$ al $17.8 \%$. Por lo que se refiere especificamente al consumo de drogas legales (alcohol y tabaco), el consumo de alcohol se ha incrementado, en tanto que el consumo de tabaco se mantiene similar al del 2003 y el nivel de consumo de ambas sustancias es prácticamente igual en hombres y mujeres. En el caso específico del abuso de alcohol se encontró un incremento de poco más del $1 \%$, de manera que actualmente $25.2 \%$ de los/as adolescentes han consumido por lo menos 5 copas en la misma ocasión durante el último mes previo al estudio.

Como se puede apreciar, el consumo de drogas se está incrementando en la población adolescente y los estudiantes no son la excepción; sin embargo los índices de consumo en la población que se mantiene escolarizada es menor cuando se le compara con otros grupos y es mayor en los jóvenes que dejan de estudiar algún tiempo (Villatoro et al., 2007).

Para combatir la problemática del consumo de drogas a nivel mundial, en un primer momento se llevaron a cabo esfuerzos considerables en comunidades y escuelas, con diversos enfoques preventivos que en su mayoria consistian en una presentación didáctica de la información para incrementar el conocimiento sobre drogas, con la expectativa de facilitar cambios en la conducta. Sin embargo, aún cuando algunos de estos programas incrementaron el nivel de conocimiento, no fueron efectivos en modificar conductas (Lantz, Jacobson, Warner, Wasserman, Pollack, Berson, et al.; OPS, 2001; Toumbourou y Stockwell, 2007).

En las últimas dos décadas se han registrado importantes avances a nivel mundial en la prevención del consumo de drogas. Los enfoques preventivos que han demostrado ser más efectivos, son los que se derivan de teorias psicosociales y se enfocan en la atención primaria y en los factores protectores que previenen la iniciación del consumo de drogas en etapas tempranas (Botvin y Kantor, 2000). Entre éstos está el enfoque de habilidades de resistencia social para prevenir el uso y/o abuso de drogas, el cual reconoce el papel de diversas influencias sociales, incluyendo el modelamiento directo de la conducta de uso de drogas y la presión del grupo de pares (Botvin y Griffin, 2004; Botvin y Griffin, 2005). Los programas desarrollados bajo este enfoque enseñan a la juventud a reconocer y resistir las presiones para usar drogas; su meta es que los estudiantes aprendan formas de evitar situaciones de alto riesgo en las cuales puedan experimentar presión para fumar, beber 0 usar drogas ilegales, así como adquirir el conocimiento que es requisito indispensable en cualquier programa preventivo (Cuijpers, 2002; Skara y Sussman, 2003).

Por otro lado, está el enfoque de fortalecimiento de habilidades que concibe al consumo de drogas como una conducta socialmente aprendida y funcional, resultado de una interacción entre factores personales y sociales. Asimismo, reconoce la importancia de los procesos de aprendizaje social, tales como el modelamiento, la imitación y el reforzamiento y considera que la juventud con pobres habilidades personales y sociales, es más susceptible a las influencias que promueven el uso de drogas; por ello hace énfasis en la importancia de enseñar habilidades personales y sociales en conjunto con habilidades de resistencia (Botvin, 2000; Organización Mundial de la Salud, OMS, 1993).

La OMS ha denominado a estas habilidades como "habilidades para la vida", que define como: "aquellas destrezas que permiten que los adolescentes adquieran las aptitudes necesarias para su desarrollo personal y para enfrentar en forma efectiva los retos de la vida diaria" (OMS, 1993) y las ha dividido en tres categorias: sociales, cognitivas y emocionales. Estas habilidades han demostrado ser eficaces en la prevención de una amplia gama de conductas de riesgo en niños y adolescentes: prevención del abuso de alcohol, tabaco y drogas ilegales; prevención del embarazo adolescente y enfermedades de transmisión sexual y resistencia a la presión de pares (Mangrulkar, Whitman y Posner 2001; OMS, 1993; OPS, 2001).

Una de las habilidades que más se ha estudiado en referencia a la prevención del consumo de drogas es la resistencia a la presión del grupo de pares, definida como la resistencia del joven ante la expresión abierta de conductas que incitan al consumo como burlas, peticiones o acciones manifiestas realizadas por los pares (Donalson, Graham, Piccinin y Hansen, 1995). Asimismo, se ha comprobado que las influencias sociales son determinantes en la iniciación del consumo de drogas, particularmente la presencia de amigos fumadores y bebedores de alcohol en la adolescencia se ha asociado a un mayor consumo de éstas (Calleja y Aguilar, 2008; Jiménez-Muro, Belmonte, Marqueta, Gargallo y Nerón, 2009). Específicamente sobre el consumo de tabaco, la literatura internacional reporta que los estudiantes no consumidores de tabaco poseen un mayor nivel de habilidad de resistencia a la presión que los estudiantes con un consumo frecuente de tabaco (De Vries, Dijk, Wetzels, Mudde, Kremers, Ariza, et al., 2006; Epstein, Bang y Botvin, 2007). Por lo que se refiere al consumo de alcohol, se ha documentado (Epstein y Botvin, 2008; Epstein, Zhou, Bang y Botvin, 2007; Latimer, Floyd, Kariis, Novotna, Exnerova y O'Biren, 2004; Londoño, Valencia, Sánchez y León, 2007) que los estudiantes no consumidores de alcohol poseen un 
mayor nivel de habilidad de resistencia a la presión que los estudiantes con un consumo frecuente de alcohol.

En el ámbito internacional se han desarrollado diversos programas escolarizados de habilidades para la vida cuyo objetivo ha sido la prevención de consumo de drogas en sus estudiantes, los cuales han incluido la habilidad de resistencia a la presión del grupo de pares como una de sus variables clave (Eisen, Zellman y Murray, 2003; Ellickson, McCaffrey, Ghosh-Dastidar y Longshore, 2003; Faggiano, Vigna-Taglianti, Versino, Zambon, Borracino y Lemma, 2008, Griffin, Botvin y Nichols, 2006; De Vries et al., 2006; Larimer y Bronce, 2002; Pentz, Mihalic y Grotpeter, 1998; Sussman, 2004; Trudeau, Spoth, Lillehoj, Redmond y Wickrama, 2003). Particularmente en Estados Unidos se identifica al "Life Skills Training" como el programa más exitoso en la prevención del consumo de drogas en estudiantes adolescentes, en el cual se ha incluido la habilidad de resistencia a la presión del grupo de pares como un componente clave desde sus inicios (Botvin y Griffin, 2005; Botvin y Kantor, 2000; Griffin, Botvin, Nichols y Doyle, 2003). No obstante, cabe destacar que las evaluaciones sobre la efectividad de estos programas se ha reportado en términos de decremento en la prevalencia de consumo de alcohol y tabaco más no sobre el incremento de la habilidad de resistencia a la presión del grupo de pares para el consumo.

En México se han llevado a cabo diversos esfuerzos preventivos que incluyen el fortalecimiento de habilidades para la vida en general y de la habilidad de resistencia a la presión en específico, también conocidas como factores de protección (Llanes y Castro, 2000). Entre los programas preventivos en México destacan: "CHIMALLI" (INEPAR, Castro, Llanes y Carreño 1999), "Promoción de estilos de comunicación asertiva" (CIJ, Flores, Arellanez, Pérez y Rodríguez, 2004), "Armando el reven" (CIJ, González, Contreras y Córdova, 2004) y "Yo quiero yo puedo" (Ruesga, Givaudan, y Pick, 2005) pero no existe evidencia sobre evaluaciones sistemáticas de estos programas ni de los instrumentos que utilizan para medir las habilidades que forman parte de su contenido.

Existe poca evidencia sobre los instrumentos utilizados para evaluar la efectividad de los programas que se reportan como exitosos y que incluyen entre sus componentes a la habilidad de resistencia a la presión, solo se identifican tres programas que si reportan los instrumentos con los que la miden: el programa "Preparing for the Drug Free Years" PDFY (Park, Kosterman, Hawkins, Haggerty, Duncan, Duncan, et al., 2000) mide la habilidad de resistencia a la presión (con 7 reactivos, $\alpha=.79$ ), por ejemplo: "Si estuvieras en una fiesta y un amigo te ofreciera una bebida alcohólica, ¿te la tomarías?"; el programa "Lions Quest- Skills for Adolescence" (Eisen et al., 2003) mide la habilidad de resistencia a la presión (con 3 reactivos, $\alpha=.92$ ) donde los reactivos se desarrollaron después de una revisión cuidadosa y de un análisis de contenido de las sesiones del programa y de juego de roles de estas sesiones; y el programa "Keepin'it REAL Currículum" (Hecht, Marsiglia, Elek, Wagstaff, Kulis, Dustman, et al., 2003) que utiliza tres estrategias de medidas de resistencia creadas especificamente para este estudio, ya que utiliza las respuestas de los estudiantes a tres reactivos para cada sustancia: alcohol, cigarros y marihuana. Los estudiantes reciben un punto por cada estrategia que señalen usar: rechazar una oferta solo diciendo "no" (negarse); dando una explicación o alguna otra excusa (explicar) y abandonando la situación (abandonar). Las calificaciones se suman para crear un escenario de tres variables que midan el número de habilidades de resistencia usadas para resistir el uso de cada sustancia. También utilizaron una escala de tres reactivos $(\alpha=.82)$ desarrollada para este estudio que midiera las intenciones de aceptar ofertas para beber alcohol, fumar cigarros o marihuana.

En contraparte existen diversos programas que reportan la medición de esta habilidad tales como el Midwestern Prevention Project (Pentz, Mihalic y Grotpeter, 1998), el proyecto "TND" (Sussman, 2004), "Yo quiero, yo puedo" (Ruesga et al., 2005) el programa de prevención de tabaco en escuelas secundarias de Helsinki (Vartiainen, Pennanen, Haukkala, Dijk, Lehtovuori, De Vries, 2007), pero ninguno reporta el instrumento con el que la miden.

Finalmente, cabe resaltar que los programas que han construido instrumentos para medir la habilidad de resistencia a la presión, lo han hecho específicamente con el grupo de pares y no han incluido a la pareja sentimental del adolescente, por lo que el presente estudio tuvo como objetivo construir y validar un instrumento que permitiera medir la habilidad de resistencia a la presión en la muestra de estudio, toda vez que en México no existen instrumentos válidos y confiables en población adolescente para medir dicha habilidad y confirmar si existen diferencias significativas en la habilidad de resistencia a la presión de pares y/o pareja en una muestra de adolescentes de bachillerato no consumidores y consumidores de alcohol y tabaco de acuerdo a su frecuencia y cantidad de consumo.

\section{MÉTODO}

\section{Participantes}

La muestra fue seleccionada de forma aleatoria y estuvo constituida por 5651 estudiantes de bachilleratos públicos de la Ciudad de México, 2637 (47.9\%) hombres y 2864 (52.1\%) mujeres, con una media de edad de 16.7 años y una desviación estándar de $1.36 ; 50.1 \%$ del turno matutino y $49.9 \%$ del turno vespertino. Referente al patrón de consumo, la edad promedio de inicio de consumo de tabaco fue de 14 años. Del total de la muestra, el 38.3\% nunca ha fumado, $28.8 \%$ si ha fumado pero no en el último mes, $19 \%$ si ha fumado de 1 a 5 días en el último mes y 13.9\% ha fumado 6 días o más en el último mes. Por lo que respecta al consumo de alcohol, 27.3\% nunca ha consumido alcohol en su vida; de los adolescentes que ya han consumido alcohol el $22.1 \%$ no ha bebido más de cinco copas completas de alcohol en una sola ocasión en el último año; $22.8 \%$ lo ha consumido por lo menos una vez en el último año, $14.6 \%$ ha tomado alcohol una vez en el último mes y $13.2 \%$ ha bebido alcohol más de 
dos veces en el último mes (datos reportados a la fecha de aplicación del estudio).

\section{Instrumento}

La elaboración y redacción de los reactivos se llevó a cabo con base en la revisión de literatura especializada en el tema de la habilidad de resistencia a la presión para consumo de drogas en adolescentes. Los reactivos fueron analizados por 6 expertos en el área. El número total de reactivos fue de 14 que exploraron la habilidad de resistencia a la presión hacia el consumo de drogas en forma de intenciones conductuales. Cabe mencionar que esta habilidad fue medida como intención conductual ya que lo importante era que todos los adolescentes respondieron lo que harían en una situación de presión aún cuando no la hubieran experimentado. Esta forma de medición se basa en la teoria de Azjen y Fishbein (1980) que señalan que las intenciones preceden a la conducta y el predictor más exacto de la conducta de una persona es por tanto su intención de llevar a cabo esa conducta. Asimismo cabe resaltar que esta habilidad se midió específicamente en relación con la pareja sentimental y/o el grupo de pares, dada la relevancia que tiene para el adolescente la pertenencia a un grupo en esta etapa de su vida. Los reactivos de la escala fueron medidos en escala Likert con cuatro opciones de respuesta: "Totalmente de acuerdo", "De acuerdo", "En desacuerdo" y "Totalmente en desacuerdo". Posteriormente se llevó a cabo el piloteo del instrumento con una muestra de 100 adolescentes estudiantes de nivel bachillerato para afinar y corregir el instrumento.

Para medir el consumo de tabaco se utilizó un indicador de riesgo donde el valor " 0 "= nunca ha fumado en la vida, "1" =sí ha fumado pero no en el último mes, "2"=sí ha fumado de 1 a 5 dias en el último mes y " 3 "=sí ha fumado 6 dias o más en el último mes. Para medir el consumo de alcohol se utilizó un indicador de riesgo donde el valor " 0 "= nunca ha bebido alcohol en la vida, "1" =sí ha bebido más de cinco copas en una sola ocasión en la vida pero no en el último año, "2"= sí ha bebido más de cinco copas en una sola ocasión por lo menos una vez en el último año y " 3 "= si ha bebido más de cinco copas en una sola ocasión una vez en el último mes y "4"= sí ha bebido más de cinco copas en una sola ocasión más de dos veces en la última semana.

\section{Procedimiento}

Se aplicó el instrumento de medición con la muestra elegida una vez aprobado el permiso por parte de las autoridades escolares en la que tuvo lugar la aplicación. Los estudiantes respondieron el cuestionario individualmente y de forma autoaplicada, en sus salones de clase, en horario preestablecido por la escuela para no afectar sus actividades académicas. Dado que el instrumento fue autoaplicado, los aspectos éticos implicados fueron el anonimato y la participación voluntaria para contestar las preguntas. El primer aspecto fue cubierto al asegurarles a los estudiantes que no se les solicitaria su nombre y los resultados serían reportados en términos globales. En relación al segundo aspecto, se les explicó a los estudiantes el objetivo de la investigación y se les preguntó si deseaban colaborar, aquellos que rehusaron a hacerlo, no fueron obligados a contestarlo. El tercer aspecto ético considerado fue la contención, toda vez que los instrumentos psicológicos pueden sensibilizar a las personas en mayor o menor grado, pero no fue necesario.

\section{RESULTADOS}

Para la validación del instrumento, se llevó a cabo el análisis de frecuencia de los reactivos y posteriormente se determinó la confiabilidad y validez de la escala. Se llevó a cabo un análisis factorial de componentes principales con rotación varimax para explorar la posibilidad de reducción de datos y obtener la validez de constructo. Se eligieron dentro de cada factor los reactivos que tuvieron un peso factorial mayor a .40 (Nunnally y Bernstein, 1995). Para cada factor obtenido se calculó la consistencia interna mediante el coeficiente de Alpha de Cronbach. El instrumento final quedó compuesto por tres subescalas de habilidades de resistencia a la presión para el consumo que explican el $45.7 \%$ de la varianza (ver Tabla 1).

Tabla 1. Análisis Factorial del instrumento de resistencia a la presión para el consumo de drogas.

\begin{tabular}{|c|c|c|c|}
\hline \multirow[t]{2}{*}{ Reactivos } & \multicolumn{3}{|c|}{ Factores } \\
\hline & 1 & 2 & 3 \\
\hline $\begin{array}{l}\text { Si mis amigos me ofrecieran un cigarro, fumaria sin } \\
\text { pensarlo. }\end{array}$ & .738 & & \\
\hline $\begin{array}{l}\text { Si mi pareja me ofreciera un cigarro, me lo fumaría aunque } \\
\text { no me gustara fumar }\end{array}$ & .690 & & \\
\hline $\begin{array}{l}\text { Me emborracharia si mis amigos acostumbraran } \\
\text { emborracharse. }\end{array}$ & .672 & & \\
\hline $\begin{array}{l}\text { Si mis amigos me invitaran a beber alcohol, aceptaria } \\
\text { aunque a mi no me gustara beber. }\end{array}$ & .583 & & \\
\hline Fumaria si mis amigos lo hicieran. & .535 & & \\
\hline $\begin{array}{l}\text { Si en una fiesta todos estuvieran bebiendo alcohol, me } \\
\text { sentiria presionado para beberlo. }\end{array}$ & .503 & & \\
\hline Fumaria si mis amigos me insistieran. & .481 & & \\
\hline Probaria las drogas, si mi pareja asi lo decidiera. & & .675 & \\
\hline $\begin{array}{l}\text { Si mi pareja me propusiera probar las drogas, le diria que sí } \\
\text { por temor a que terminara conmigo. }\end{array}$ & & .672 & \\
\hline $\begin{array}{l}\text { Si mi pareja insistiera en que yo fumara, defenderia mi } \\
\text { derecho de no hacerlo. }\end{array}$ & & .482 & \\
\hline $\begin{array}{l}\text { Si mi pareja me pidiera que lo acompañara a emborracharse, } \\
\text { le diría claramente que no deseo hacerlo. }\end{array}$ & & & 631 \\
\hline $\begin{array}{l}\text { Si mis amigos me presionaran para probar las tachas (1), } \\
\text { me opondria. }\end{array}$ & & & .615 \\
\hline Aunque mi pareja fumara, yo no fumaria. & & & .596 \\
\hline $\begin{array}{l}\text { Si mis amigos me invitaran a un cigarro, les diría que no } \\
\text { quiero fumar. }\end{array}$ & & & .551 \\
\hline
\end{tabular}

Porcentaje total de varianza explicada: $45.7 \%$

(1) Tachas = pastillas 
Tabla 2. Coeficientes alpha de Cronbach de los tres factores que conforman el instrumento de resistencia a la presión para el consumo de drogas.

\begin{tabular}{ccc}
\hline Factor & Número de reactivos & Alpha \\
\hline Aceptación de la presión de amigos & 7 & .80 \\
Aceptación de la presión de pareja & 3 & .54 \\
Resistencia a la presión de amigos & 4 & .57 \\
\hline
\end{tabular}

En la tabla 2 se observan los tres factores obtenidos en la escala de la habilidad de resistencia a la presión: 1) aceptación de la presión de amigos 2) aceptación de la presión de pareja 3 ) resistencia a la presión de amigos y/o pareja.

A continuación se presentan las definiciones de cada factor obtenido:

Aceptación de la presión de amigos: Se refiere a la intención de ceder a la presión de amigos para consumir drogas.

Aceptación de la presión de pareja: Se refiere a la intención de ceder a la presión de la pareja para consumir drogas.

Resistencia a la presión de amigos y/o pareja: Se refiere a la intención de resistir a la presión de amigos y/o pareja para consumir drogas.

Para identificar si existían diferencias en los adolescentes no consumidores y consumidores de tabaco y alcohol con respecto a su habilidad de resistencia a la presión, se realizó un análisis de varianza de una sola vía y se aplicó la prueba Post Hoc Scheffé para observar cual de los grupos marcaba las diferencias. Cabe mencionar que las medias de cada factor se calcularon con base en la suma de los reactivos que componen cada factor divididos entre el número de reactivos.
Los resultados mostraron diferencias estadisticamente significativas en los puntajes de cada factor de la escala de acuerdo a la frecuencia de consumo de tabaco. Los resultados de la prueba Scheffé indicaron que el grupo que marca la diferencia en la habilidad de resistencia a la presión $(F=129.34, p<.001)$ es el de los adolescentes que nunca han consumido tabaco con respecto a los otros grupos (ver tabla 3). Estos estudiantes obtuvieron puntajes más altos en el factor de resistencia a la presión de amigos y pareja en comparación con los adolescentes que han fumado en el último mes, sobre todo aquellos que han fumado 6 o más dias. Por lo que respecta a la aceptación de la presión de amigos $(F=148.315, p<.001)$ y pareja $(F=18.806, p<$ .001), el puntaje fue más bajo en los grupos que consumen con más frecuencia.

En cuanto al consumo de alcohol, los resultados mostraron diferencias estadisticamente significativas en los puntajes de cada factor de la escala de acuerdo a la frecuencia de consumo. Los resultados de la prueba Scheffé mostraron que el grupo de adolescentes que nunca ha consumido alcohol es el que marca la diferencia en la habilidad de resistencia a la presión con respecto a los otros grupos ( $F=74.389, p<.001)$ (ver tabla 4$)$. Los estudiantes que nunca han consumido una copa completa de alcohol en su vida obtuvieron puntajes más altos en el factor de resistencia a la presión de amigos y pareja en comparación con los adolescentes que han consumido más de cinco copas de alcohol en el último mes, sobre todo aquellos que han bebido con más frecuencia (más de dos veces en la última semana). Por lo que respecta a la aceptación de la presión de amigos ( $F$ $=86.646, p<.001)$ y pareja $(F=20.565, p<.001)$, el puntaje fue más bajo en los grupos que consumen con más frecuencia.

Tabla 3. ANOVA de una sola vía de las habilidades de resistencia por consumo de tabaco.

\begin{tabular}{|c|c|c|c|c|c|c|c|c|}
\hline & \multicolumn{2}{|c|}{$\begin{array}{c}\text { Nunca } \\
\text { han fumado }\end{array}$} & \multicolumn{2}{|c|}{$\begin{array}{l}\text { No han fumado } \\
\text { en el último mes }\end{array}$} & \multicolumn{2}{|c|}{ De 1 a 5 días en el último mes } & \multicolumn{2}{|c|}{$\begin{array}{l}\text { De } 6 \text { o más dias } \\
\text { en el último mes }\end{array}$} \\
\hline & M & D.S. & M & D.S. & M & D.S. & M & D.S. \\
\hline Aceptación a la presión de amigos & $1.33^{*}$ & 0.47 & 1.47 & 0.49 & 1.69 & 0.57 & 1.66 & 0.52 \\
\hline Aceptación de presión de pareja & $1.20^{*}$ & 0.44 & 1.24 & 0.45 & 1.30 & 0.48 & 1.31 & 0.47 \\
\hline $\begin{array}{l}\text { Resistencia a la presión de amigos } \\
\text { y/o pareja }\end{array}$ & $3.63^{*}$ & 0.52 & 3.52 & 0.53 & 3.29 & 0.60 & 3.28 & 0.58 \\
\hline
\end{tabular}

${ }^{*} p<.001$ Scheffé

Tabla 4. ANOVA de una sola vía de las habilidades de resistencia por consumo de alcohol.

\begin{tabular}{|c|c|c|c|c|c|c|c|c|c|c|}
\hline & & & \multicolumn{8}{|c|}{ Si han bebido más de 5 copas } \\
\hline & \multicolumn{2}{|c|}{$\begin{array}{c}\text { Nunca } \\
\text { han tomado alcohol }\end{array}$} & \multicolumn{2}{|c|}{$\begin{array}{c}\text { Pero no en el último } \\
\text { año }\end{array}$} & \multicolumn{2}{|c|}{ Una vez en el último año } & \multicolumn{2}{|c|}{ Una vez en el último mes } & \multicolumn{2}{|c|}{$\begin{array}{c}\text { Más de } 2 \text { veces en la } \\
\text { última semana }\end{array}$} \\
\hline & M & D.S. & M & D.S. & M & D.S. & M & D.S. & M & D.S. \\
\hline $\begin{array}{l}\text { Aceptación a la presión de } \\
\text { amigos }\end{array}$ & $1.35^{*}$ & 0.48 & 1.40 & 0.48 & 1.53 & 0.50 & 1.61 & 0.54 & 1.72 & 0.57 \\
\hline Aceptación de presión de pareja & $1.20^{*}$ & 0.44 & 1.21 & 0.43 & 1.24 & 0.43 & 1.31 & 0.51 & 1.36 & 0.50 \\
\hline $\begin{array}{l}\text { Resistencia a la presión de ami- } \\
\text { gos y/o pareja }\end{array}$ & $3.60^{*}$ & 0.55 & 3.59 & 0.52 & 3.46 & 0.55 & 3.35 & 0.58 & 3.24 & 0.59 \\
\hline
\end{tabular}

${ }^{*} p<.001$ Scheffé 


\section{DISCUSIÓN}

Uno de los problemas de la investigación sobre habilidades para la vida realizada en México, ha sido la escasa medición de éstas; por ello construir una escala de habilidad de resistencia a la presión en adolescentes mexicanos resultaba una tarea prioritaria. La aportación de este estudio se traduce primeramente en un instrumento con tres subescalas para medir la habilidad de resistencia a la presión para el consumo de drogas en adolescentes mexicanos escolarizados, con niveles aceptables de confiabilidad de acuerdo al número de reactivos de cada una de ellas.

La mayoria de los estudios (Eisen et al., 2003; Hecht et al., 2003; Park et al., 2000) que presentan la escala con la que miden esta habilidad hacen referencia solo a los amigos y no a la pareja, situación que sí se contempla en esta escala, lo cual la hace más completa en tanto que se incluye a otra persona importante para los jóvenes en esta etapa de su vida.

Por otra parte, cabe destacar que la habilidad de resistencia a la presión fue medida en forma de intención conductual, ya que no era posible evaluar la conducta que reflejara la habilidad como tal en todos los adolescentes. En este sentido, Azjen y Fishbein (1980) mencionan que las intenciones preceden a la conducta y el predictor más exacto de la conducta de una persona es por tanto su intención de llevar a cabo esa conducta. Por ello, la medición de que la intención está presente lleva a considerar que muy probablemente en el futuro y cuando las demandas situacionales lo faciliten, los adolescentes que manifiestan intenciones conductuales hacia el consumo de drogas, ya sea a favor o en contra, instrumentarán estas conductas. Es así que la escala de resistencia a la presión con sus tres factores permite medir la aceptación y el rechazo de presión por parte de amigos y/o pareja para el consumo de drogas.

Por lo que se refiere a las diferencias encontradas entre los adolescentes no consumidores y consumidores de tabaco y alcohol con respecto a su habilidad de resistencia a la presión, este estudio pone de relieve la importancia que posee el principal grupo de referencia en la adolescencia, el cual está conformado por el grupo de pares y la pareja. Los adolescentes que nunca han consumido tabaco ni alcohol en su vida o que no lo han consumido en el último mes, obtuvieron puntajes más altos en su habilidad de resistencia a la presión que los adolescentes que han fumado en el último mes, sobre todo aquellos que han fumado y bebido alcohol con más frecuencia; en contraparte, los adolescentes que presentan un consumo frecuente de tabaco y alcohol en el último mes poseen una mayor aceptación de la presión de su grupo de pares y/o pareja para el consumo de tabaco y alcohol.

Los hallazgos de esta investigación confirman en México lo reportado en estudios anteriores en otros paises (De Vries et al., 2006; Epstein et al., 2007; Epstein y Botvin, 2008; Epstein et al., 2007; Latimer et al., 2004), en el sentido de que la habilidad de resistencia a la presión es uno de los factores más importantes para la prevención del consumo de drogas en adolescentes, o como lo sería en este caso específico, de consumo de tabaco y alcohol.

Si como se ha reportado en estudios anteriores, la presencia de amigos cercanos consumidores de drogas es un factor predictor del consumo en adolescentes (Calleja y Aguilar, 2008; Jiménez-Muro et al., 2009); se puede concluir a través de la investigación citada (De Vries et al., 2006; Epstein et al., 2007; Epstein y Botvin, 2008; Epstein et al., 2007;; Latimer et al., 2004) que el desarrollo de la habilidad de resistencia a la presión del grupo de pares y/o pareja puede retrasar el inicio del abuso de drogas. Por ello un entrenamiento en esta habilidad es recomendable para la promoción y mantenimiento del estado de salud en la niñez y adolescencia (OMS, 1993).

En cuanto a las limitaciones de este estudio, se tienen las presentes en la construcción de todo instrumento psicológico de medición: la influencia de la deseabilidad social, la escasez de estudios de validación externa sobre las autoevaluaciones (los datos obtenidos con escalas en cuanto relatos de desempeño necesitan ser validados por indicadores directos de ese rendimiento) (Prette y Prette, 2002) y el carácter situacional de la habilidad de resistencia a la presión, es decir la posibilidad de sesgos dependiendo de cómo el adolescente interpreta el contexto de la situación descrita en los reactivos. Sin embargo, resulta pertinente recordar que la construcción de instrumentos válidos y confiables para medir fenómenos psicológicos es el primer paso para el diseño, implementación y evaluación adecuada de programas preventivos acordes con las necesidades de cada población y en función de los recursos existentes (Observatorio Mexicano en tabaco, alcohol y otras drogas, 2003). La escala derivada de esta investigación puede ser utilizada para medir la habilidad de resistencia a la presión de pares y/o pareja relacionada con la prevención del consumo de drogas en adolescentes mexicanos escolarizados. No obstante, se recomienda incrementar el número de reactivos en las escalas de aceptación de la presión de pareja y resistencia a la presión de amigos y/o pareja para elevar el nivel de confiabilidad de dichas escalas. Finalmente, para futuras investigaciones se sugiere medir otras habilidades reportadas en la literatura como relacionadas con la prevención del consumo de drogas en adolescentes.

Por último, queda claro que si se desea promover conductas saludables y libres de riesgo en población adolescente, los programas escolarizados deben incluir un contenido informativo claro y detallado, que permita a su vez revertir las creencias asociadas y facilitar la adquisición de habilidades especificas relacionadas con la conducta meta, como lo sería en este caso, la habilidad de resistencia a la presión identificada como relacionada con la prevención del consumo de drogas en adolescentes (OPS. 2001). El siguiente paso sería desarrollar programas de prevención de adicciones basados en un enfoque de habilidades para la vida que permita evaluar estas conductas. 


\section{AGRADECIMIENTOS}

Este estudio se realizó gracias al apoyo de la Universidad Nacional Autónoma de México, la Secretaría de Desarrollo Institucional, el Programa Transdisciplinario en Investigación y Desarrollo para Facultades y Escuelas, a través de su Macroproyecto Desarrollo de Nuevos Modelos para la Prevención y el Tratamiento de Conductas Adictivas.

\section{REFERENCIAS}

Azjen, I., y Fishbein, M. (1980). Understanding attitudes and predicting social behavior. Englewood Cliffs, NJ: Prentice-Hall.

Botvin, G. (2000). Preventing drug abuse in schools: Social and competence enhancement approaches targeting individual level etiological factors. Addictive Behaviors, 25, 887-897.

Botvin, G., y Griffin, K. (2004). Prevention science, drug abuse prevention and Life Skills Training: Comments on the state of the science. Journal of Experimental Criminology, 1, 63-78.

Botvin, G., y Griffin, K. (2005). Life Skills Training: Empirical findings and future directions. Journal of Primary Prevention, 25, 211232.

Botvin, G., y Kantor, L. (2000). Preventing alcohol and tobacco use through life skills training theory, methods and empirical finding. Alcohol Research and Health, 24, 250-257.

Calleja, N., y Aguilar, J. (2008). Por qué fuman las adolescentes: Un modelo estructural de la intención de fumar. Adicciones, 20, 387-394.

Castro, M.E., Llanes, J., y Carreño, S. (1999). Chimalli, un modelo de prevención de riesgos psicosociales. Psicología Iberoamericana, 7, 14-23.

Cuijpers, P. (2002). Effective ingredients of school based drug prevention programs. A systematic review. Addictive Behaviors, 27, 1009-1023.

De Vries, H., Dijk, F., Wetzels, J., Mudde, A., Kremers, S., Ariza, C., et al. (2006). The European Smoking Prevention Framework Approach (ESFA): Effects after 24 and 30 months. Health Education Research, 21, 116-132.

Donaldson, S., Graham, I., Piccinin, A., y Hansen, W. (1995). Resistance skills training and onset of alcohol use: Evidence for beneficial and potentially harmful effects in public schools and in private catholic schools, Health Psychology, 14, 291-300.

Eisen, M., Zellman, G., y Murray, M. (2003). Evaluating the Lions "Skills for adolescence" drug education program. Second year behavior outcomes. Addictive Behaviors, 28, 883-897.

Ellickson, L. (1998). Preventing adolescent substance abuse: Lessons from the Project ALERT Program. New York: Russell Sage Foundation.

Ellickson, L., McCaffrey, D., Ghosh-Dastidar, B., y Longshore, D. (2003). New inroads in preventing adolescent drug use: Results from a large-scale trial of Project ALERT in Middle Schools. American Journal of Public Health, 93, 1830-1836.

Encuesta Nacional de Adicciones (ENA) (2002). México: Secretaría de Salud.

Epstein, J., Bang, H., y Botvin, G. (2007). Which psychosocial factors moderate or directly affect substance use inner-city adolescents? Addictive Behaviors, 32, 700-730.
Epstein, J., y Botvin, G. (2008). Media resistance skills and drug skill refusal techniques: What is their relationship with alcohol use among inner-city adolescents? Addictive Behaviours, 33, 528537.

Epstein, J., Zhou, X., Bang, H., y Botvin, G. (2007). Do Competence Skills Moderate the Impact of Social Influences to Drink and Perceived Social Benefits of Drinking on Alcohol Use Among Inner-City Adolescents? Preventive Science, 8, 65-73

Faggiano, F., Vigna-Taglianti, F., Versino, E., Zambon, A., Borracino, A. y Lemma, P. (2008). School-based prevention for illicit drugs use: A systematic review 2008. Preventive Medicine, 232-246.

Flores, L., Arellanez, H., Pérez, I., y Rodríguez, K. (2004). Evaluación formativa de un programa de promoción de estilos de comunicación asertiva para el afrontamiento de la presión social para el uso de sustancias psicoactivas con estudiantes de educación media (informe de investigación 01-14, publicación interna). México: Centros de Integración Juvenil.

González, J., Contreras, S., y Córdova, J. (2004). Evaluación de resultados del módulo "Armando el Reven" entre adolescentes y adultos jóvenes en situación del alto riesgo (informe de investigación 04-17, publicación interna). México: Centros de Integración Juvenil.

Griffin, K., Botvin, G., Nichols, T., y Doyle, M. (2003). Effectiveness of a universal drug abuse prevention program for youth at risk for substance abuse initiation. Prevention Medicine, 36, 1-7.

Griffin, K., Botvin, G., y Nichols, T. (2006). Effects of a school drug abuse prevention program for adolescents on HIV risk behaviors in young adulthood. Prevention Science, 7, 103-112.

Hecht, M. L., Marsiglia, F. F., Elek, E., Wagstaff, D. A., Kulis, S., Dustman, $P_{.}$, et al. (2003). Culturally grounded substance use prevention: An evaluation of the keepin' it REAL curriculum. Prevention Science, 4, 233-248.

Jiménez-Muro Franco, A., Beamonte San Agustín, A., Marqueta Baile, A., Gargallo Valero, P., y Nerín de la Puerta, I. (2009). Consumo de drogas en estudiantes universitarios de primer curso. Adicciones, 21, 21-28.

Lantz, M., Jacobson, D., Warner, E., Wasserman, J., Pollack, A., Berson, J., et al. (2000). Investing in youth tobacco control: A review of smoking prevention and control strategies. Tobacco Control, 9, 47-63.

Larimer, M., y Bronce, J. (2002). Identification, prevention and treatment: A review of individual focused strategies to reduced problematic alcohol consumption by college students. Journal of Studies on Alcohol, 14, 148-163.

Latimer, W., Floyd, I., Kariis, T., Novotna, G., Exnerova, P., y O'Biren, M. (2004). Peer and sibling substance abuse: Predictors of substance use among adolescents in Mexico. Public Health, 15, 225-232.

Londoño, C., Valencia, S., Sánchez, L., y León, V. (2007). Diseño del cuestionario resistencia a la presión de grupo en el consumo de alcohol (CRPG). Suma Psicológica, 14, 271-285.

Llanes, J., y Castro, M. E. (2000). Prevención cientifica de la farmacodependencia en México. Instituto de Educación Preventiva y Atención de Riesgos, A.C.

Mangrulkar, L., Whitman, C. V., y Posner, M. (2001). Enfoque de habilidades para la vida para un desarrollo saludable de niños y adolescentes. Washington, DC: Organización Panamericana de la Salud. 
Nunnally, J. C., y Bernstein, I. H. (1995). Teoría Psicométrica. México: McGraw- Hill.

Observatorio Mexicano en tabaco, alcohol y otras drogas, (2003). México: Secretaria de Salud.

Organización Mundial de la Salud (OMS) (1993). Enseñanza en los colegios de las habilidades para vivir. Ginebra: Organización Mundial de la Salud.

Organización Panamericana de la Salud (OPS) (2001). Enfoque de habilidades para la vida para un desarrollo saludable de niños y adolescentes. Washington, DC: Autor.

Park, J., Kosterman, R., Hawkins, D., Haggerty, K., Duncan, T., Duncan, S., et al. (2000). Effects of the "Preparing for the Drug Free Years" Curriculum on Growth in Alcohol Use and Risk for Alcohol Use in Early Adolescence. Prevention Science, 1, 125-138.

Pentz, M., Mihalic, S., y Grotpeter, J. (1998). Blueprints for violence prevention. Book one: The Midwestern Prevention Project. Boulder, CO: Center for the Study and Prevention of Violence.

Prette, Z., y Prette, A. (2002). Psicología de las habilidades sociales: terapia y educación. México: Manual moderno.

Ruesga, C., Givaudan, M. y Pick, S. (2005). Yo quiero, yo puedo: un modelo educativo de competencias psicosociales para México. México: Cuadernos de la Secretaria de Educación Pública.

Skara, S., y Sussman, S. (2003). A review of 25 long-term adolescent tobacco an other drug use prevention program evaluations. Preventive Medicine, 37, 453-474.

Toumbourou, J., y Stockwell, T. (2007). Interventions to reduce harm associated with adolescent substance use. Adolescent Health, 4, 1-11.

Trudeau, L., Spoth, R., Lillehoj, T., Redmond, S., y Wickrama, P. (2003). Effects of a prevention intervention on adolescent substance abuse initiation, expectancies and refusal intentions. Prevention Science, 4, 109-122.

Vartiainen, E., Pennanen, M., Haukkala, A., Dijk, F., Lehtovuori, R., y De Vries, H. (2007).The effects of a three-year smoking prevention program in secondary schools in Helsinki. European Journal of Public Health, 17, 249-256.

Villatoro, J., Gutiérrez, M., Quiroz, N., Moreno, M., Gaytán, L., Gaytán, F., et al. (2007). Encuesta de Consumo de Drogas en Estudiantes 2006. México, D.F.: Instituto Nacional de Psiquiatria Ramón de la Fuente Muñiz. 